\title{
Adolescentes y jóvenes: desde las conductas de riesgo a la inclusión social
}

\author{
Amando Vega \\ Departamento de Didáctica y Didáctica y Organización Escolar, \\ Universidad del País Vasco UPV/EHU \\ <amando.vega@ehu.es>
}

\section{Pello Aramendi}

Departamento de Didáctica y Didáctica y Organización Escolar, Universidad del País Vasco UPV/EHU

\section{Segundo Garín}

Departamento de Didáctica y Didáctica y Organización Escolar, Universidad del País Vasco UPV/EHU

Biztanle gazteenen arrisku-egoerak eta arriskujokabideak berrikusi eta eskola-porrota horien eragile gisara ulertu ostean, aurkezten dira ikerkuntzaren arlotik landutako esku hartzeko aukerak. Nabarmentzen dira hezkuntza inklusiboa eta beronen eskakizunak, eta horien artetik gailentzen da testuinguruan kokatzea, bereiziki kontuan harturik eskola-geletan gertatzen dena, eta betiere ikuspegi inklusibo batetik testuingurua hobetzen saiatuz. Gaur eguneko krisialdiari eraginkortasunez aurre egiteko, derrigorrezkoa da garapenerako alde guztiak kontuan hartzen dituen haurtzaro eta nerabezaroaren aldeko politika iraunkorren apustu egitea: osasun fisiko, psikologikoa eta harremanen artekoa, hezkuntza, aisialdia, segurtasuna, familia eta ingurugiroa.

HITZ-GAKOAK:

nerabeak, gazteak, osasuna, arrisku-jokabideak.
En este trabajo, tras revisar la literatura sobre las situaciones de riesgo en la población más joven y sobre sus conductas de riesgo, y valorar el fracaso escolar como el detonante de esta situación, se presentan las diferentes propuestas de intervención que surgen desde el ámbito de la investigación. Se resalta en ese sentido la importancia de la educación inclusiva y sus exigencias, entre las que destaca su contextualización, con especial atención a lo que ocurre en las aulas y en centros educativos concretos, tratando de mejorar su contexto cercano con la mirada puesta en la inclusión social. Para afrontar de forma eficaz una crisis como la actual, es imprescindible apostar de forma sostenida por políticas a favor de la infancia y adolescencia que contemplen todos los aspectos esenciales de su desarrollo: salud física, psicológica y relacional, educación, ocio y tiempo libre, seguridad, familia y medio ambiente.

\section{PALABRAS CLAVE:}

adolescentes, jóvenes, salud, conductas de riesgo, inclusión, integración social. 


\section{Introducción}

Los problemas de los adolescentes y jóvenes nunca están al margen de la sociedad en la que viven. Por esto al analizar sus conductas de riesgo resulta imprescindible contextualizar sus vidas al mismo tiempo que comprender su proceso vital. Sus conductas son un reflejo de la sociedad en la que viven.

Hoy es evidente que la Estrategia Europa 2020, el crecimiento sostenible e inclusivo y los objetivos de reducción de la pobreza están cada vez más subordinados al motor político dominante de la estabilización del euro, a la reducción del déficit y la deuda principalmente a través de una consolidación fiscal que resulta socialmente dañina y que está claramente generando más pobreza, exclusión y desigualdad, como denuncia la Carta de EAPN... (2011). La crisis económica, social y moral que vive nuestra sociedad se refleja también en el colectivo adolescente y joven. Valores como la competitividad y la ganancia fácil priman sobre valores como la solidaridad y el respeto (Eresta y Delpino, 2012).

No dar respuestas coherentes a las necesidades de adolescentes y jóvenes, no sólo refuerza sus problemas sino que también augura un futuro incierto para su desarrollo personal y social que no es otro que el de la exclusión con sus profundas raíces y diferentes caras visibles. La sociedad a la que ellos y ellas pertenecen, por otra parte, pierde al mismo tiempo un capital humano, del que no puede prescindir para su desarrollo equilibrado y saludable. Lo resalta la Comisión Europea (2011) cuando sostiene que la mejora de los resultados educativos de los adolescentes responde tanto a los objetivos de "un crecimiento inteligente", con la mejora de las capacidades, como a los objetivos de "un crecimiento integrador", al enfrentarse a factores básicos de riesgo como el desempleo y la pobreza.

Hay que reconocer que sacar a luz las contradicciones no significa resolverlas, como señala Bourdieu (1999: 559), para denunciar de forma explícita el dejar hacer:

Lo seguro, en todo caso, es que nada es menos inocente que el laissez-faire: si es verdad que la mayoría de los mecanismos económicos y sociales que están en el origen de los sufrimientos más crueles, en especial los que regulan el mercado laboral y el mercado escolar, son difíciles de frenar o modificar, lo cierto es que toda política que no aproveche plenamente las posibilidades, por reducidas que sean, que se ofrecen a la acción, y que la ciencia puede ayudar a descubrir, puede considerarse culpable de no asistencia a una persona en peligro.

\section{Efectos de la crisis en la población más joven}

España es uno de los países que ha registrado un mayor aumento de la población en riesgo de pobreza y exclusión social, al pasar del $23,4 \%$ en 2009 al 25,5\% en 2011, incrementándose en algo más de un millón de personas, hasta alcanzar un total de 11,6 millones, según la ultima memoria del Consejo Económico y Social (Observatorio Español de la Discapacidad, 2012). El debilitamiento del Estado del bienestar se une a un empeoramiento de las condiciones de la calidad de vida y a un aumento de las desigualdades que queda patente en que la renta de la población con mayores ingresos fue 6,9 veces superior a la de la población con ingresos más bajos en 2010, mientras que en la Unión Europea la diferencia, siendo importante, se reduce y es cinco veces superior. Los recortes, por otra parte, pueden 'cronificar' la situación de áreas como la sanidad, la educación y los servicios sociales.

Pero veamos con detalle el alcance de esta situación social para la población más joven. El estudio realizado por la Fundación FOESSA (Laparra y Pérez, 2010) ya puso de manifiesto el importante crecimiento de las tasas de pobreza y precariedad como consecuencia de la crisis económica, que afecta de forma especial a los sectores más vulnerables. En torno al $25 \%$ de la población ha visto cómo empeoraba su situación en términos de integración social, y los sistemas de protección social, pese al incremento en el número de hogares beneficiarios, no han podido compensar los efectos del desempleo.

Según el estudio de Clua-Losada et al. (2011) sobre la infancia en riesgo de pobreza o exclusión social en España, aquellos sectores que estaban en riesgo siguen estándolo, mientras que algunos colectivos han empeorado. Por otra parte, si bien se nota una gran inseguridad en cuanto a recursos, el efecto de los recortes todavía no se ha manifestado de manera directa. Pero el problema llegará, cuando los programas actuales no cuenten con una financiación segura, ni con un proyecto de financiación futura, y sus posibilidades empiezan a verse reducidas.

Unicef (2012) completa esta información cuando destaca que, en España, casi 2,2 millones de niños viven en pobreza relativa. Por primera vez en la historia de este país, los niños se han convertido en el colectivo más afectado por la crisis económica. La pobreza infantil, estabilizada durante años en torno al $24 \%$ de la población menor de edad, ha superado el $26 \%$. Casi 205.000 niños más viven en hogares con unos ingresos por debajo del umbral de la pobreza. En cuanto a persistencia e intensidad de la pobreza, la pobreza crónica ha aumentado en un $53 \%$ en tres años.

Este diagnóstico del momento actual no puede dejar indiferente a ningún educador que se sienta comprometido con los derechos de la infancia, sabiendo las consecuencias negativas que esta situación puede tener sobre el desarrollo educativo de los 


\section{Continúa presente entre los educadores la vieja tendencia de poner en manos de policías, jueces, psicólogos o trabajadores sociales las conductas problemáticas de los jóvenes}

menores, con deriva en unos casos hacia conductas problemáticas.

Sin embargo, continúa presente entre los educadores la vieja tendencia de poner en manos de policías, jueces, psicólogos o trabajadores sociales las conductas problemáticas que suelen aparecer entre la población más joven. Se olvida que la población más joven, con la educación inclusiva, tiene pleno derecho, no sólo a estar presente en las escuelas y en otros servicios educativos, sino a recibir en el entorno ordinario la atención educativa que necesitan. Son, ante todo, sujetos de educación, por su consideración de menores, y por ello tienen que aprender a vivir y a convivir (Aramendi y Ayerbe, 2009) desde las instituciones educadoras. Y si la adolescencia trae consigo una serie de cambios en diferentes ámbitos (físicos, intelectuales, emocionales) que van a suponer una auténtica revolución en todas las áreas de la vida del joven o la joven, esta situación se agrava en la sociedad actual que con la llamada 'crisis' provoca nuevas dificultades en los adolescentes y jóvenes, sobre todo, en contextos de pobreza y marginación.

Existe, por otra parte, el profundo peligro que señala Nussbaum (2010: 4) de la crisis mundial en materia de educación, prácticamente inadvertida, que puede llegar a ser mucho más perjudicial para el futuro de la democracia:

se están produciendo cambios drásticos en aquello que las sociedades democráticas enseñan a sus jóvenes, pero se trata de cambios que aún no se sometieron a un análisis profundo. Sedientos de dinero, los Estados nacionales y sus sistemas de educación están descartando sin advertirlo ciertas aptitudes que son necesarias para mantener viva a la democracia. Si esta tendencia se prolonga, las naciones de todo el mundo en breve producirán generaciones enteras de máquinas utilitarias, en lugar de ciudadanos cabales con la capacidad de pensar por sí mismos, poseer una mirada crítica sobre las tradiciones y comprender la importancia de los logros y los sufrimientos ajenos.

Llama la atención la preocupación del mundo del cine por la problemática de la población adolescente con dificultades de adaptación bajo el paraguas de los 'niños salvajes'. La directora de una de estas pelícu- las puede ayudarnos en esta reflexión con su comprometido prólogo de la novela Alex (Yagüe, 2012). Los 'niños salvajes' están mal educados porque a la sociedad actual no le importa la educación del individuo, que sea verdaderamente libre, sino sólo que sirva a unos intereses. Hay gente 'necesaria' y gente 'que no hace falta': muchos de los niños, los menos dotados, los más pobres, los que no sirven para ser portadores de los actuales 'valores', basados sobre todo en éxito y dinero, no son necesarios. ¿Para qué perder el tiempo educándolos?

¿Qué hacemos, entonces, con los malos? Es una pregunta que debiera hacernos también nosotros, pero no a raíz de un problema concreto que hayamos podido tener en nuestra relación con ellos, sino desde la serena comprensión de su situación personal y social, del trato recibido en su familia, de la atención educativa ofrecida por la escuela, de la acogida que pueda tener en su comunidad y en los servicios públicos que ésta presta.

Aunque, la mayoría de los jóvenes están sanos, como señala la OMS (2010), cada año se registran más de 2,6 millones de defunciones en la población de 15 a 24 años. Un número considerable de jóvenes sufren enfermedades que reducen su capacidad para crecer y desarrollarse plenamente. Y un número aún mayor adoptan comportamientos que ponen en peligro su salud presente y futura. Por esto, la promoción de las prácticas saludables en la adolescencia y la adopción de medidas para proteger mejor a los jóvenes frente a los riesgos para su salud son fundamentales para el futuro de la infraestructura sanitaria y social de los países y para prevenir la aparición de problemas de salud en la edad adulta. Los principales problemas de salud son el consumo de tabaco, la falta de actividad física, la obesidad, las relaciones sexuales sin protección y la exposición a la violencia.

\section{Las conductas de riesgo en la juventud}

En nuestro país no faltan investigaciones sobre las conductas de riesgo en la adolescencia para comprender el alcance del problema. El estudio de Meneses et al. (2010) sobre los comportamientos de riesgo -consumo de drogas, violencia, seguridad vial y relaciones sexuales- en la población adolescente de tres comunidades autónomas -Andalucía, Madrid y País Vasco- aporta datos relevantes sobre estas conductas.

Las chicas (32\%) manifiestan haberse emborrachado en mayor medida que los chicos $(25,9 \%)$, y lo mismo cabe decir del grupo de 'blancos' en comparación con el de 'otros'. En cuanto al consumo diario de tabaco, son también las chicas (11,3\%) las que presentan porcentajes más altos en consumo diario, en comparación con los chicos (7,4\%). En cuanto a las drogas ilegales aparecen consumos inferiores a los de otros estudios de ámbito nacional. No hay diferencias a destacar en consumo de cannabis por sexo, pero sí por etnia, siendo los jóvenes del grupo 
de 'otros' los que registran mayor consumo. Destacan, por otra parte, los comportamientos de riesgo relacionados con la conducción de ciclomotores y el consumo de alcohol u otras drogas. En este sentido, cuatro de cada diez jóvenes encuestados manifiestan haber ido a mayor velocidad de la permitida y un $18 \%$ afirman haberse retado en la carretera. Además, un $7 \%$ dice haber montado, alguna o muchas veces, en motocicleta después de haber consumido alcohol, y el $4,5 \%$ indica haber consumido hachís u otras drogas antes de conducir. Estos comportamientos de riesgo fueron realizados sobre todo por varones y por el grupo autoidentificado como 'otros'.

En la descripción de los hábitos de salud de la población joven de Gipuzkoa (Martín Roncero et al., 2010), la mitad de la población joven es sedentaria durante el tiempo libre, condicionado por diversos factores estructurales. El uso del preservativo en las relaciones sexuales ocasionales es bajo, ya que el $40 \%$ de las personas jóvenes de la CAPV afirma no haberlo usado en el último año. Las tasas de interrupción voluntaria del embarazo son más altas en Gipuzkoa respecto al resto de territorios, pero más bajas que en el resto del Estado.

\section{Los principales problemas} de salud son el consumo de tabaco, la falta de actividad física, la obesidad, las relaciones sexuales sin protección y la exposición a la violencia

En relación al alcohol, su consumo en la población joven es similar al del resto de la población salvo los fines de semana, en los que el consumo aumenta en las personas jóvenes por tratarse de un componente esencial del modelo de ocio juvenil vigente. El patrón de consumo de tabaco, referido por el $30 \%$ de las personas jóvenes, se adquiere durante la juventud y se mantiene, en términos generales, durante la vida adulta. Este consumo aparece ligeramente mayor en las mujeres, y aumenta a medida que se desciende la clase social.

Las lesiones por accidentes de tráfico son la principal causa de mortalidad y discapacidad entre la población joven, aunque se han reducido en los últimos años. En Gipuzkoa las personas jóvenes presentan unas tasas de accidentalidad mayores que en el resto de territorios, especialmente en el caso de las mujeres. La mortalidad por accidentes de tráfico es mayor en hombres que en mujeres, lo que posiblemente esté relacionado con el modelo de masculinidad hegemónico.

Respecto a los hábitos alimentarios, parece estar produciéndose un alejamiento por parte de la población joven de una dieta variada y equilibrada, un aumento del consumo de alimentos grasos y azucarados y un descenso del de frutas, verduras y pescado. Además, un $20 \%$ de personas afirma no desayunar. El género, el nivel socioeconómico, así como la situación familiar y la emancipación residencial influyen en el tipo de hábitos alimentarios que se adquieren.

Son datos que nos acercan al mundo complejo de las conductas problemáticas de infancia y juventud en una sociedad en cambio y que coinciden en sus aspectos más genéricos con los obtenidos en nuestras ultimas investigaciones sobre las drogas en los Programas de Cualificación Profesional Inicial (Vega y Aramendi, 2012) y sobre la educación para la salud en los centros de ESO (Vega et al., 2012).

Estos resultados no pueden deberse solamente a características individuales o de personalidad, sino también a factores sociales como la diversidad cultural o de género, contextos socioeconómicos o políticas relacionadas con la población más joven, como se resalta en el estudio realizado en Gipuzkoa. Se trata de cuestiones a tener en cuenta, pues, en todos los centros educativos, ya que estas conductas problemáticas, más allá de las implicaciones personales, se convierten en una carga añadida al trabajo ya por sí complejo de los profesores (Otero et al., 2012).

Habrá que tener en cuenta, al mismo tiempo, las respuestas que reciben este tipo de conductas desde las diferentes instituciones públicas y privadas para valorar su alcance, sin olvidar sus posibles implicaciones negativas no sólo para los menores sino también para el conjunto de la sociedad a corto y a largo plazo, como lo hace Ararteko (2011) en su informe sobre las Infancias vulnerables. Son menores en grave riesgo de desprotección o en desamparo; población adolescente que comete algún delito y es objeto de medidas judiciales; alumnado con necesidades educativas especiales o específicas; salud mental infanto-juvenil; convivencia en el ámbito escolar; problemática de los y las adolescentes gays, lesbianas, bisexuales y transexuales.

Como una situación específica de exclusión social se puede recordar la que viven los alumnos y alumnas de los Programas de Cualificación Profesional Inicial, dadas sus circunstancias personales y sociales. Sin apoyo educativo adecuado tendrán muchas dificultades para construir su proyecto vital y laboral. Comprender los factores personales y sociales que condicionan sus vidas, permitirá responder a sus necesidades educativas a caballo entre la formación personal y la iniciación profesional. La tara educativa es ante todo una labor de mediación educativa que les permitirá obtener la titulación de la ESO y la cualificación profesional (Vega, y Aramendi, 2011).

Otra situación de riesgo es la pueden vivir las persona con una sexualidad diferente. Según el informe del Ararteko (2008), hay carencias de socialización 
en las relaciones sexo-afectivas, el ambiente gay comercial es la única salida para muchos, la falta educación sexual específica con lo que implica de vulnerabilidad y de riesgo, las instituciones públicas prácticamente no les tienen en cuenta para nada. Los institutos y la adolescencia se convierten entonces en un tiempo y un espacio donde se exacerba la homofobia.

Los hijos de familias inmigrantes, por su parte, tienen dificultades para cubrir las necesidades alimentarias, van a la escuela sin desayunar, se duchan con poca frecuencia porque no tienen agua en casa o no pueden pagar medicinas, entre otras (Quiroga y Alonso, 2011).

Las conductas de riesgo exigen una lectura social para ir más allá de sus manifestaciones más próximas y ofertar respuestas a corto y a largo plazo más coherentes con sus necesidades, tareas que parecen quedar marginadas en una sociedad más pendiente de los beneficios del mercado que de los derechos de las personas. La consecuencia es, como señala el Informe Conjunto al Comité de Derechos Económicos, Sociales y Culturales (2012), la notable regresividad en la protección y el disfrute de los derechos económicos, sociales y culturales que podrían suponer las políticas adoptadas por el Estado en respuesta a la crisis económica y financiera; la desigual distribución de recursos en el país que da lugar a diferencias y a vulneraciones en el disfrute los derechos económicos, sociales y culturales dependiendo de la zona geográfica; y la ausencia de medidas de protección social para paliar los efectos de la crisis sobre los derechos de personas o poblaciones en situación de mayor vulnerabilidad, como las mujeres, los trabajadores migrantes, las personas con discapacidad, la comunidad gitana y la infancia. Preocupa que las medidas de austeridad fiscal que está adoptando el gobierno para hacer frente a la crisis no sólo se limitan a la reducción de inversión social hasta extremos que afectan el contenido esencial de los derechos reconocidos en el Pacto Internacional de Derechos Económicos, Sociales y Culturales (ONU; 1966), sino que, para ajustarse al reducido margen presupuestario modifican y derogan garantías legales de los derechos sociales.

\section{El fracaso escolar como detonante}

El fracaso y el absentismo escolar que tanto preocupan hoy en Europa no dejan de ser una manifestación más de las carencias educativas existentes, que si, por una parte, roban a muchos menores sus posibilidades de crecimiento personal y social, no facilitan, por otra, el desarrollo de una sociedad justa e inclusiva (Vega, 2011). Así, los adolescentes en conflicto con la ley son adolescentes con una experiencia educativa en términos de fracaso escolar, con expulsiones y ausencias del sistema educativo, en definitiva, excluidos de éste y seccionados de uno de los mecanismos de integración social más importantes en la sociedad (Uceda y Pérez, 2010).
Se entiende así que la Organización para la Cooperación y el Desarrollo Económico (OCDE, 2012) urja a sus países miembros a invertir en educación como solución a largo plazo de la crisis económica, ante el coste que tiene para la sociedad y los individuos el fracaso escolar de sus estudiantes. La cara más visible del fracaso escolar es precisamente el abandono de la escuela. La OCDE precisa que en 2010 había unos 15 millones de jóvenes parados en sus países miembros, cuatro millones más que en 2007, así como que sólo la mitad de quienes habían dejado la escuela tenían un empleo, lo que les hace más dependientes de las ayudas sociales y sanitarias públicas. Por contra, los sistemas educativos con el rendimiento más alto en los países de la OCDE combinan calidad con equidad. Por esto, reducir el fracaso escolar ahorra gastos a largo plazo.

\section{Las conductas de riesgo exigen una lectura social para ir más allá de sus manifestaciones más próximas}

Pero, como señalan Fernández Enguita et al. (2011) tras su investigación, el fracaso y el abandono escolar no son fruto de una decisión puntual, sino consecuencia de un proceso de progresiva desvinculación de la escuela que tiene su origen mucho tiempo antes de que el problema se manifieste. La repetición de curso es un fenómeno que empieza en la educación primaria. El fracaso escolar se acentúa en la educación secundaria obligatoria, con un $28,4 \%$ de alumnos que abandonan la ESO sin graduarse. Un $14,8 \%$ de los abandonos se produce antes de los 16 años, la edad que marca el fin de la educación obligatoria. En bachillerato sólo consiguen pasar de curso el $76,7 \%$ de los alumnos de primero y logran el título, al aprobar segundo, sólo el 73,2\% de los alumnos. En el caso de los ciclos formativos de grado medio, el $48,9 \%$ de sus estudiantes lleva al menos un año de retraso respecto a lo esperable para su edad. El número de graduados en bachillerato o ciclos formativos de grado medio alcanza en España el 62\%.

El riesgo de fracaso escolar, de acuerdo con indicadores procedentes del estudio PISA, aparece asociado a ciertas variables, como el género, el estatus socioeducativo de los padres o el origen étnico. Los chicos presentan tasas de fracaso más elevadas que las chicas en todos los niveles educativos. Así, un $41 \%$ de los chicos de 15 años tiene alto riesgo de fracaso, mientras que en las chicas es sólo de un $29 \%$. La ocupación y el nivel educativo de los padres influyen en el riesgo de experimentar fracaso escolar. Así, el $45 \%$ de alumnos de clases trabajadoras presenta un riesgo elevado, frente a un $23 \%$ entre los de las clases medias. En el caso de los estudios de los padres, el $20 \%$ de alumnos con padres universitarios tiene riesgo de fracaso, mientras que este riesgo afecta al $63 \%$ de los alumnos con padres sin estudios y al $42 \%$ 
de alumnos con padres que cursaron sólo estudios primarios. Un $55 \%$ de los alumnos inmigrantes tienen riesgo de fracasar frente a un $34 \%$ de alumnos españoles. En los estudiantes inmigrantes de segunda generación (nacidos en España de padres inmigrantes), el riesgo de fracasar se sitúa en valores intermedios (41\%) [Fernández Enguita et al., 2011].

\section{El abandono escolar}

aparece como resultado final de un progresivo proceso de desvinculación de la escuela

El abandono, pues, aparece como resultado final de un progresivo proceso de desvinculación de la escuela, como se puede ver tras el análisis de una muestra de expedientes de alumnos que abandonó prematuramente la escuela. Esta desvinculación comienza mucho antes del momento de abandono y se manifiesta precisamente en forma de problemas disciplinarios, absentismo o bajas calificaciones, por lo que se refiere a la escuela, mientras que fuera del ámbito escolar aparecen diferentes conductas de riesgo como el consumo de drogas, conductas violentas, entre otras.

Las variables del ámbito familiar determinan en un alto grado el riesgo de fracaso escolar del alumno, recuerdan Calero et al. (2010). Así, los hijos de padres económicamente activos y/o de categoría socioeconómica 'cuello blanco cualificado', aquellos alumnos y alumnas cuyos hogares están mejor dotados de recursos materiales educativos, y los que hacen un mejor uso de dichos recursos tienen una menor probabilidad de fracaso escolar. Atención especial merecen la condición de inmigrante y el idioma utilizado en el hogar. Los alumnos nacidos en el extranjero en familias de origen inmigrante tienen un mayor riesgo de fracaso escolar. Sin embargo, dicho riesgo se va reduciendo a medida que avanza el proceso de adaptación al país de acogida. En todo caso, los resultados asociados a las variables linguísticas subrayan la importancia de disponer, de cara a estudios futuros, de mayor información sobre el perfil de los alumnos de origen inmigrante.

Los resultados de aprendizaje están hoy determinados por las condiciones sociales, económicas y culturales de las familias. Pero esas condiciones han cambiado, como recuerda Tedesco (2011), por lo que es necesario recuperar los resultados de los estudios recientes sobre las nuevas formas que asumen las desigualdades, donde juega un papel fundamental la construcción social de situaciones de exclusión, de ruptura de los vínculos con la sociedad, de ausencia de proyecto y de perspectivas de futuro. Es necesario superar el enfoque tradicional, como resalta el autor citado, donde el esfuerzo se ponía en analizar la contribución de la educación a la equidad social, para postular la necesidad de un mínimo básico de equidad y cohesión social como condición necesaria para que sea posible un proceso educativo exitoso.

Conviene recordar, por otra parte, las perversiones del sistema macro y micro recordadas por Fernández Enguita (2011) refiriéndose a una cultura institucional y profesional, hasta cierto punto transmutada en social, que produce buena parte de los casos de fracaso y abandono. Apunta, en primer lugar, la idea generalizada de que no todo el mundo vale para estudiar, expresada hoy en el abuso de la figura punitiva de la repetición, carente de cualquier lógica y probadamente ineficaz. En segundo lugar, el cierre de cualquier vía para los no graduados en ESO. Lo que llamamos educación 'general' no es sino una unilateral tradición sesgada y ranciamente académica, apegada a la herencia de las artes liberales; es decir, que el rechazo del trabajo manual está siempre implícito y, a veces, hasta explícito en la práctica pedagógica dominante. De hecho, España está muy bien situada en el concierto internacional por número $y$ tasa de graduados en Bachillerato y en estudios superiores, pero no en la postobligatoria en su conjunto, pues quiebra espectacularmente en Formación Profesional, porque es la propia educación común la que aleja a los alumnos de ella.

No hay que olvidar, pues, los grandes retos que tiene el sistema educativo en general, y de forma especial, la ESO y las cuestiones pendientes de respuesta: políticas socioeducativas dirigidas al alumnado de origen social menos favorecido; infraestructuras y recursos humanos; políticas educativas que ayuden a los adolescentes a una transición de calidad entre etapas; currículum más flexible, con menos asignaturas, pero más contenido y actualidad; la participación de las familias y de nuevos perfiles profesionales en el apoyo de la docencia, en una etapa especialmente compleja para las personas. Los cambios en la población que se atiende deben venir acompañados de transformaciones profundas en el diseño y la implementación de los circuitos de protección (Quiroga y Alonso, 2011).

Es lo que también pide la Unesco (2012) cuando pone de manifiesto la "apremiante" necesidad de invertir en educación para que los jóvenes adquieran competencias. Frente al promedio del 14 por ciento de jóvenes de países de la Unión Europea que no llega más allá del primer ciclo de la enseñanza Secundaria (ESO), en España esta cifra se duplica y uno de cada tres alumnos abandona los estudios de Secundaria. Por esto, pide la atención a la adecuación de la enseñanza secundaria al mundo del trabajo, al mismo tiempo que advierte que, con la crisis económica, las posibilidades de empleo de los jóvenes han disminuido, siendo particularmente afectados aquellos con niveles de educación inferiores. También alerta de que tras estas cifras de paro juvenil se oculta el hecho de que los jóvenes dejan de buscar trabajo porque "creen no poder encontrarlo", es decir, existe un colectivo que "ni estudia, ni trabaja, ni busca activamente trabajo". 


\section{Recomendaciones no faltan}

El Comité de Protección Social de la Comisión Europea (2011) propone una respuesta global, tras constatar los efectos de la crisis económica actual en la evolución de la exclusión social. Se necesita priorizar la atención temprana en áreas de la salud y la educación infantil; mejorar la disponibilidad de servicios de atención a la infancia de calidad; promover la participación de los progenitores en el mercado laboral y, por último, garantizar las ayudas destinadas a las familias con niños. Hace falta el establecimiento de unas políticas de empleo y educación bien diseñadas, así como el saneamiento de las finanzas públicas y la reforma de los sistemas de protección social para asegurar su sostenibilidad, protegiendo a los sectores más vulnerables. También considera esencial la implantación de estrategias activas de inclusión que combinen el apoyo a los ingresos, el acceso al mercado de trabajo y a los servicios de salud y sociales para prevenir la exclusión, así como el aumento de la eficiencia del gasto social, con especial atención a las necesidades de las mujeres y las personas jóvenes.

No se niega, por esto, que se haya avanzado mucho en la respuesta educadora dirigidas a los adolescentes y jóvenes. De todas formas, conviene ser conscientes tanto de las carencias existentes como de los retos que plantea la sociedad desbocada en las que vivimos, incapaz de ofrecer un futuro esperanzador a la población joven. Habrá que tener en cuenta los estudios que analizan la práctica educativa y las respuestas institucionales dadas tanto en el ámbito local y autonómico, como en el más amplio, nacional e internacional, sobre todo aquellos desarrollados por instituciones independientes.

Los resultados académicos de los alumnos de familias desfavorecidas están vinculados a las condiciones de ingreso de los alumnos a la escuela. El desarrollo cognitivo básico, que se produce en los primeros años de vida y está vinculado a una sana estimulación afectiva, buena alimentación y condiciones sanitarias adecuadas, y, por otra, una socialización primaria adecuada mediante la cual los niños adquieren los rudimentos de un marco básico que les permita incorporarse a una institución especializada distinta a la familia, como la escuela. Las políticas al respecto deberían, por ello, atacar las desigualdades en términos de recursos culturales disponibles en las familias, para favorecer un proceso de socialización primaria destinado a promover un adecuado desarrollo cognitivo básico. De ahí la importancia de la atención temprana para promover igualdad y equidad educativa y romper así el círculo vicioso de padres pobres-hijos pobres (Tedesco, 2011). Esto significa invertir en las familias y en la primera infancia. Existen numerosas evidencias que indican el escaso poder compensador de las desigualdades que tiene la educación formal si interviene una vez que estas ya han sido creadas.
No faltan propuestas sobre aspectos específicos como el realizado por Aróstegui et al. (2010), que estudia el papel de las instituciones vascas respecto a los consumos de drogas en la adolescencia. El estudio muestra que, a pesar de que existe una relación muy extendida entre buena parte de los adolescentes y algunas drogas -especialmente el tabaco, el alcohol y el cannabis-, se dan también grandes variaciones entre los distintos grupos, que será necesario tener en cuenta para un correcto abordaje de las cuestiones que se plantean.

Según expone el informe, hay dos grandes necesidades de mejora: por un lado, la coordinación entre todas las instancias que trabajan en el campo del consumo de drogas y la adolescencia; y por otro, el impulso a la prevención selectiva, dirigida específicamente a determinados sectores de la población adolescente. Hay que aclarar que se habla de prevención y no de asistencia, puesto que el estudio se centra en menores que no han desarrollado problemas de adicción. Y las actuaciones deben tomarse en una doble dirección: identificar los factores de riesgo para el inicio del consumo, y también detectar y neutralizar los factores que favorecen el mantenimiento del hábito, diseñando intervenciones dirigidas a reducirlos. De acuerdo con las demandas de los colectivos profesionales más cercanos a la población adolescente, es prioritario avanzar en el campo de la prevención selectiva. El informe señala los diversos contextos en los cuales debe actuar la prevención selectiva: el educativo, el familiar y el comunitario. En el ámbito escolar, por ejemplo, es preciso proporcionar a los centros los instrumentos pedagógicos que sirvan para superar los factores de riesgo relacionados con el consumo de drogas. El medio escolar tiene mucho que aportar en la prevención. Por ello, es necesario poner en marcha iniciativas de apoyo al profesorado, a través de formación y asesoramiento. Pero también son los propios profesores y profesoras quienes tienen que hacer un esfuerzo añadido para cumplir con los objetivos de la prevención selectiva, puesto que, además de formarse en cuestiones básicas relacionadas con las drogas, deberían hacerlo para poder diagnosticar potenciales situaciones de riesgo y vulnerabilidad.

Por lo que se refiere al ámbito familiar, existe un conflicto entre las necesidades de las familias, que a menudo se sienten desamparadas y desbordadas, y su capacidad para tolerar críticas o sugerencias en lo que respecta a la educación de sus hijas e hijos. Es importante señalar que, en el caso de familias con mayor vulnerabilidad, mantener un contacto más o menos regular con otros recursos, como los servicios sociales, así como la comunicación y coordinación entre las distintas entidades, podría servir para ofrecerles la prevención adecuada.

Los espacios de ocio juvenil permiten aproximarse a determinados colectivos no escolarizados ni detectados por la red asistencial, que manifiestan conductas de riesgo y consumo de sustancias ilegales. Los recursos de ayuda, sin embargo, siguen diseñándose para las demandas de la mayoría, 
no coinciden con las de la juventud conflictiva, y, por tanto, siguen transmitiéndoles un mensaje de inadaptación que no contribuye a la prevención, sino que incluso puede aumentar los factores de riesgo en este colectivo.

Por todo esto, se recomienda la coordinación interinstitucional entre los diferentes agentes que intervienen en la población menor de edad, y que su trabajo conjunto se centre en el desarrollo de la prevención, dirigida específicamente a determinados grupos que resultan más vulnerables por diversos factores, tanto en el ámbito educativo, como en el familiar y en el del tiempo libre, en el que se producen la mayoría de los consumos adolescentes. Además de ello, hace especial hincapié en que se eviten las incoherencias en el discurso social y se dé cabida en él a las voces de los propios adolescentes, potenciando su papel activo como agentes de prevención. La intervención con la adolescencia y la juventud en situación de riesgo y exclusión requiere que participen distintos sectores profesionales y sociales.

Clua-Losada et al. (2011), por su parte, consideran como los principales retos en la lucha contra la pobreza o exclusión social de la infancia la formación, la prevención y la coordinación de los servicios que atienden a estos colectivos. Ven necesario, por otra parte, invertir en investigaciones sociales donde el menor sea la unidad de análisis, no necesariamente su hogar o familia. E insisten finalmente en la necesidad de una aproximación a la infancia que tome el niño o niña como eje vertebrador de cualquier política y programa. Únicamente a partir de un enfoque de derechos de la infancia, que supere la perspectiva familiar, las necesidades y los derechos de la infancia conseguirán no sólo visibilidad, sino también un avance en la dirección necesaria para el desarrollo de un Estado del bienestar.

El efecto negativo de las diferencias de renta sobre el bienestar, nos obliga a comprometernos con la infancia y amortiguar la "austeridad mal entendida", como señala Hernán (2012):

- Dirigiendo las acciones hacia familias con menores rentas con programas de conciliación de la vida familiar y laboral, servicios de atención primaria de salud y pediatría, equipos de bienestar social infantil y de tratamiento familiar, actividades de los centros de protección de menores, acogimiento y adopción, y la promoción de la salud y el buen trato a la infancia.

- Potenciando el conocimiento sobre el estado de la infancia y las intervenciones para mejora de su calidad de vida e investigando sobre la sociedad del conocimiento, los programas educativos que funcionan, las conductas favorables para la salud y otros asuntos prevalentes o problemas de salud infantil.

- Desarrollando la calidad y los recursos de educación formal en nuestras comunidades tanto los de educación primaria y secundaria, como las escuelas infantiles.

- Preparando una base segura para crecer saludablemente, facilitando información, asesoramiento y formación a madres, padres, tutores, educadores, profesionales afines y otros sectores implicados de la sociedad, para concienciarnos de la importancia que tiene el desarrollo los primeros años de vida y la creación de condiciones idóneas para el crecimiento saludable.

Por su parte, Unicef (2012) ve imprescindible mirar más allá del corto plazo, analizando detenidamente las posibles consecuencias presentes y futuras de las decisiones que se tomen en cada momento. Además de proteger la inversión en la infancia, aboga por una mejora de la calidad, la eficiencia y la coordinación de las políticas destinadas a la infancia. Y aporta como alternativas: poner a los niños y sus familias en el núcleo de las decisiones políticas, mejorar la coordinación, la eficacia y la coherencia en la gestión de la salud, la educación, la protección de la infancia y la lucha contra la pobreza y elaborar un Plan Nacional contra la Pobreza Infantil.

\section{6. ¿Qué puede aportar el sistema educativo ante esta complicada y exigente situación?}

Aunque a lo largo de este escrito, ya se han sugerido diferentes aportaciones que el sistema educativo puede desarrollar, conviene resaltar algunos aspectos que permitan delimitar con visión amplia y comprometida la misión del sistema educativo con relación a la infancia y la adolescencia. Se trata de resaltar los principios que han de sustentar todo el esfuerzo para la inclusión social de la población joven en situación de riesgo.

La educación es un derecho fundamental de la persona. Forma parte del conjunto de derechos económicos, sociales y culturales posteriores al desarrollo de los derechos civiles y políticos y, por ello, considerados de segunda generación, como recuerdan Bonal y Tabarini (2012). La Declaración Universal de los Derechos Humanos, de 1948, la Convención de los Derechos de la Infancia de Naciones Unidas, de 1989 , y un buen número de pactos internacionales reconocen el derecho de la persona a acceder a una educación que garantice su pleno desarrollo personal.

Urge una redefinición ampliada del derecho a la educación como apuntan los autores citados, teniendo en cuenta la insuficiencia de la escolarización obligatoria, los requisitos de acceso al mercado de trabajo, la competencia por las credenciales educativas o la importancia de la escolarización temprana. Pero no faltan deficiencias en disponibilidad, acceso, adaptación y calidad del sistema educativo, que siempre perjudican más a los más vulnerables. La suma de estas vulneraciones de derechos, junto con la ampliación de las dimensiones 
del derecho a la educación, componen un escenario preocupante para los próximos años, desde el punto de vista de la distribución de las oportunidades educativas. Hay que recordar, además, que en la equidad educativa reside la legitimidad de un Estado del bienestar cada vez más debilitado por la crisis económica. Los retos de la educación para el siglo XXI son, pues, enormes, sobre todo para unos sistemas educativos a los que se va exigir cada vez más esfuerzo para garantizar la igualdad de oportunidades de los ciudadanos.

El sistema educativo, pues, desde su compromiso con la educación inclusiva y el desarrollo de contextos saludables y el marco de la justicia curricular (Torres, 2010) tendrá que poner en marcha los medios que convengan para prevenir y tratar las conductas problemáticas. Por esto la educación inclusiva -es decir, una educación eficaz y de alta calidad para todos los niños y jóvenes-constituye el mayor reto que deben enfrentar los sistemas educativos del mundo entero, ya se trate de los países en desarrollo o de los países desarrollados. Se trata de acoger la diversidad de todos los educandos. Ello exige la adaptación del currículum, medidas organizativas flexibles y recursos humanos y materiales para atender a los distintos ritmos, estilos de aprendizaje y a las distintas habilidades.

La institución escolar es la mejor garantía del derecho a la educación. Pero su adecuado funcionamiento compete tanto a la comunidad escolar como a quienes tienen responsabilidades políticas y de gestión sobre el sistema escolar. Como también la academia, desde una perspectiva crítica, para ayudar en la comprensión, explicación y aportación de criterios para contribuir a que la dinámica escolar cotidiana funcione de modo apropiada, prestando especial atención a la producción de desigualdades que acompaña inevitablemente a la cotidianeidad escolar.

En esta línea, son de interés las propuestas de Eresta y Delpino (2012) quienes, tras estudiar las aspiraciones y los modelos de los adolescentes actuales y mostrar aspectos de la vida de los adolescentes que resultan significativos en su formación, para estructurar sus identidades individuales y colectivas, plantean como necesidades, entre otras:

- Mejorar los mecanismos que flexibilicen el sistema educativo, con el propósito de ofrecer itinerarios abiertos y programas efectivos de compensación educativa, así como actividades dirigidas a la prevención del fracaso escolar.

- Enriquecer la orientación y calidad de los programas educativos en su vinculación con el empleo. Favorecer la ampliación y mejora de la calidad de las acciones de orientación profesional.

- Patrocinar foros de debate entre adolescentes respecto de varias de las cuestiones que actualmente constituyen fuentes de su preocupación, que les faciliten la búsqueda de alternativas de atención a sus demandas.
- Construir y ampliar espacios de debate y de formación de las familias en torno a la atención de las necesidades de los jóvenes y a la transmisión de valores.

- Emplazar al poder público para que lleve adelante determinadas campañas que difundan modelos coherentes con el tipo de ciudadano democrático al que aspiramos.

- Velar porque los medios de comunicación dirijan a los jóvenes mensajes y modelos que coadyuven a su desarrollo.

En cuanto a esta última propuesta, el estudio de Sevillano et al. (2009) deja constancia de que los programas muestran mensajes que invitan a los adolescentes a presentar ciertos comportamientos poco apropiados, peligrosos, violentos, consumo de sustancias adictivas, práctica sexual temprana, indiscriminada y promiscua, confusión de romance, sexo y fantasía, no dan pie a la contemplación ni a la reflexión respecto de sí mismos y de sus características personales.

Los centros escolares, por otra parte, deben vincularse a las estructuras territoriales donde se incardinan y promover la participación, implicación y compromiso de los estudiantes en y con su comunidad local, y ello como una opción de intervención socioeducativa que apunta a componentes nucleares de su formación y desarrollo como personas y ciudadanos. Sea cual sea el tipo y modalidad de proyecto que se adopte, se hace necesario no sólo la conformidad y coordinación de todas las instancias implicadas, o una formación de profesores y estudiantes en clave comunitaria, sino también unos requerimientos de planificación, gestión, metodología y evaluación, a fin de garantizar adecuados niveles de calidad educativa en los procesos y resultados (Cieza, 2010).

\section{Con la mirada en el futuro}

Hoy que tanto se habla de la educación inclusiva y que con demasiada facilidad se pone la etiqueta de inclusivo a cualquier servicio dedicado a persona excluidas, sin pensar en las consecuencias a largo plazo de sus 'aportaciones', viene muy bien escuchar a profesionales que desde hace tiempo luchan por hacer realidad el compromiso inclusivo. Porque el sistema educativo mantiene sus estructuras, currículum, organización de los centros, prácticas escolares y una formación docente sin tener en cuenta la diversidad. Urge, por esto, comprender las características específicas de todos y cada uno de los alumnos, así como los factores personales y sociales que condicionan sus vidas, para poder responder a sus necesidades educativas. Y, al mismo tiempo, disponer de recursos humanos, materiales y funcionales adecuados.

La Conferencia Internacional de Educación celebrada en Ginebra en 2008 bajo el lema 'La educación inclusiva: el camino hacia el futuro’ (Unesco, 2008: 3), no puede ser más clara al respecto: 
De la atención que reciban los niños y adolescentes dependerá, en buena medida, la conducta social adulta de quienes hoy son menores de edad

La educación inclusiva es un proceso que entraña la transformación de las escuelas y otros centros de aprendizaje para atender a todos los niños, tanto varones como niñas, a alumnos de minorías étnicas, a los educandos afectados por el VIH y el sida y a los discapacitados y con dificultades de aprendizaje. El proceso educativo se lleva a cabo en muchos contextos, tanto formales como no formales, en las familias y en la comunidad en su conjunto. Por consiguiente, la educación inclusiva no es una cuestión marginal, sino que es crucial para lograr una educación de calidad para todos los educandos y para el desarrollo de sociedades más inclusivas.

No basta con formular leyes específicas, como ha sido el caso de España en los años ochenta y posteriores (Toboso et al., 2012), sino que se debe mantener un compromiso profundo con la educación inclusiva bajo el liderazgo de la administración y la coordinación de todos los servicios relacionados con el alumnado. Las políticas para la inclusión educativa deben atender a todos los componentes del sistema educativo necesitados de mejora: la formación y cualificación del profesorado y otros profesionales de la educación, el cambio de las dinámicas de programación educativa y el diseño curricular, la modificación de los contextos en los que se incluye a los alumnos, la evaluación y financiación del sistema, así como el cambio en las actitudes y la lucha contra los estereotipos. Al mismo tiempo, se debe pensar la inclusión educativa de manera local y situada, prestando atención a lo que ocurre en las aulas y centros concretos, tratando de mejorar este contexto para avanzar hacia cambios más globales y sistémicos.
Son necesarias al mismo tiempo políticas a favor de la Infancia con un carácter estratégico, si se desea elevar los niveles de calidad de vida y de bienestar de una sociedad. De la atención que reciban los niños y adolescentes dependerá, en buena medida, la conducta social adulta de quienes hoy son menores de edad. Por ello, para afrontar de forma eficaz una crisis como la actual, es imprescindible apostar de forma sostenida por políticas a favor de la infancia que contemplen todos los aspectos esenciales de su desarrollo: salud física, psicológica y relacional, educación, ocio y tiempo libre, seguridad, familia y medio ambiente. Como también favorecer los análisis compartidos de la realidad de la infancia y adolescencia, con un carácter preventivo y con una especial atención a los colectivos más desfavorecidos y excluidos; involucrar a todos los agentes sociales de la comunidad y dar respuesta inmediata al inicio de los procesos de exclusión con criterios de calidad, eficacia y eficiencia (Aranda et al., 2012).

La 'cohesión social' es fruto de la capacidad de un sistema social, económico y político de promover la autonomía y la participación social de los ciudadanos, la creación de redes sociales e institucionales que generen capital social y favorezcan la inclusión social; y su contribución a la materialización de los derechos sociales en su más amplio sentido (Rodríguez Cabrero et al., 2011). En el caso de la población más joven, la no atención educativa de sus necesidades personales y sociales no deja de ser el robo de su infancia (Vega, 2011).

La sociedad, pues, tiene ante los niños, adolescentes y jóvenes una urgente responsabilidad, que debe ser asumida por todas las instituciones, pero de forma especial por todas aquellas con responsabilidad educadora. Por ello, es necesario conocer con la mayor profundidad posible el sentido y el alcance de sus conductas de riesgo con todos sus condicionantes (empleo, vivienda), para poder desarrollar las respuestas educativas preventivas o terapéuticas que convengan en cada situación. Las conductas de riesgo no dejan de ser una manifestación más de una problemática social de fondo en las que diferentes factores familiares, escolares y sociales condicionan la vida de la población joven en riesgo de exclusión social. 
ARAMENDI, P.; y AYERBE, P. (2009): “Aprender a convivir: un reto en la Educación Secundaria Obligatoria”, Educación y Diversidad, nㅜ3, págs. 61-105.

ARANDA, G. et al. (2012): “Ciudades amigas de la infancia: una aportación a la cohesión social desde las políticas locales de infancia y adolescencia”, Revista Española Tercer Sector, n-0 20, págs. 11-23.

ARARTEKO (2011): Infancias vulnerables. Informe extraordinario de la institución del Ararteko al Parlamento Vasco. Informes extraordinarios, Vitoria-Gasteiz, Ararteko.

- (2008): Adolescentes y jóvenes lesbianas gays, transexuales y bisexuales: dificultades y rechazos en su desarrollo personal, en sus relaciones y en su socialización, VitoriaGasteiz, Ararteko [<http://www.ararteko.net/ RecursosWeb/DOCUMENTOS/1/o_1819_1.pdf)].

ARÓSTEGUI, E. et al. (2010): El papel de las instituciones vascas respecto a los consumos de drogas de la adolescencia, serie Informes Extraordinarios, Vitoria-Gasteiz, Ararteko [<http://www.ararteko.net/RecursosWeb/ DOCUMENTOS/1/1_1871_3.pdf〉].

BONAL, X.; TABARINI-CASTELLANI, A; y VERGER, A. (2012): Globalización y educación: textos fundamentales, Bueno Aires, Miño y Dávila.

BOURDIEU, P. (1999): Razones prácticas. Sobre la teoría de la acción, Barcelona, Anagrama.

CALERO, J.; WAISGRAIS, S.; y CHOI, A. (2010): "Determinantes del riesgo de fracaso escolar en España: una aproximación a través de un análisis multinivel aplicado a PISA 2006", Revista de Educación, no extraordinario 1, págs. 225-256.
CASAS, F.; MONTSERRAT, C.; y MALO, S. (2010): La educación y los jóvenes ex tutelados, Girona, Universitat de Girona [<http://tcru.ioe.ac.uk/yippee/ Portals/1/reportinspanish.pdf〉].

COMITÉ DE DERECHOS ECONÓMICOS, SOCIALES Y CULTURALES (2012): Informe Conjunto al Comité de Derechos Económicos, Sociales y Culturales. Examen del $5^{0}$ Informe Periódico de España, Madrid, Comité de Derechos Económicos, Sociales y Culturales [khttp://www2.ohchr. org/english/bodies/cescr/docs/ngos/ JointSubmission19NGOs_Spain_CESCR48_ sp.pdf $>$ ].

CIEZA, J. A. (2010): "El compromiso y la participación comunitaria de los centros escolares. Un nuevo espacio-tiempo de intervención socioeducativa”, Pedagogía Social. Revista Interuniversitaria, nํ17, págs. 123-136.

CLUA-LOSADA, M.; SESÉ, A.; y TUR, M. (2011): "Infancia y exclusión social en España: realidades y retos a partir de la crisis", Zerbitzuan, $\mathrm{n}-50$, págs. 71-84 [khttp://dx.doi.org/10.5569/11347147.50.06>].

COMISIÓN EUROPEA (2011): Abordar el abandono escolar prematuro: una contribución clave a la agenda Europa 2020, Bruselas, Comisión Europea.

COMITÉ DE PROTECCIÓN SOCIAL DE LA UNIÓN EUROPEA (2011): La dimensión social de la Estrategia Europea 2020, Luxemburgo, Comisión Europea.

EAPN (2011): Carta de EAPN a los Primeros Ministros y Presidentes de Gobierno, Madrid, EAPN [khttp://eapn.es/ARCHIVO/documentos/ recursos/1/1088_2011-carta_las_los_PM_ Encuesta_Nacional_de_Crecimiento.pdf`]. 
ERESTA, M. J.; y DELPINO, M. A. (2012): Adolescentes de hoy. Aspiraciones y modelos, Madrid, Ministerio de Sanidad, Servicios Sociales e Igualdad.

FERNÁNDEZ ENGUITA, M. et al. (2011): Fracaso y abandono escolar en España, col. Estudios Sociales, no 29, Barcelona, Fundación La Caixa [khttp://multimedia.lacaixa.es/lacaixa/ ondemand/obrasocial/pdf/estudiossociales/ vol29_completo_es.pdf)].

HERNÁN, M. (2012): “Crisis, austeridad y políticas de infancia. Los niños son las primeras víctimas de los recortes", El País, 7-5-2012.

LAPARRA, M.; y PÉREZ, B. (2010): El primer impacto de la crisis en la cohesión social en España, Madrid, Fundación FOESSA.

MARHUENDA, F. (2011): "Presentación. Dimensiones didácticas y organizativas que sustentan la dinámica escolar”, Revista de Educación, nํㅜ 356, págs. 17-37.

MARÍ-KLOSE, P. et al. (2010): Infancia y futuro. Nuevas realidades, nuevos retos, col. Estudios Sociales, nํㅜ 30, Barcelona, Fundación La Caixa [<http://obrasocial.lacaixa.es/StaticFiles/Static Files/7af433edaa007210VgnVCM1000000e8cf1 oaRCRD/es/vol3o_es.pdf)].

MARTÍN RONCERO et al. (2010): La promoción de la salud en la población joven de Gipuzkoa: ampliando la visión a los determinantes sociales de la salud, Donostia-San Sebastián, Diputación Foral de Gipuzkoa.

MENESES, C. et al. (2010): "Adolescencia, consumo de drogas y comportamientos de riesgo: diferencias por sexo, etnicidad y áreas geográficas en España", Trastornos Adictivos, vol. $11, \mathrm{n}^{0} \mathrm{1}$, págs. 51-63.

NUSSBAUM, M. (2010): La crisis silenciosa [rhttp:// literalmagazine.blogspot.com/2010/10/lacrisis-silenciosa.htmls].

OBSERVATORIO ESTATAL DE LA DISCAPACIDAD (2012): EI CES alerta sobre el incremento de las desigualdades y la exclusión social en España, Olivenza, Observatorio Estatal de la Discapacidad [<http://www.observatoriodeladiscapacidad. es/informacion/noticia/19202〉].

OCDE (2012): Equity and Quality in Education. Supporting Disadvantaged Students and Schools, Londres, OECD Publishing.

OMS (2010): Riesgos para la salud de los jóvenes, serie Nota Descriptiva, $\mathrm{n}-345$ [ [www.who.int/ mediacentre/factsheets/fs345/es/index. html>].

ONU (1966): Pacto Internacional de Derechos Económicos, Sociales y Culturales [/http://www.acnur.org/ t3/fileadmin/scripts/doc.php?file=biblioteca/ pdf/o014'].
OTERO, J. M. et al. (2012): Estrés laboral y burnout en profesores de Enseñanza Secundaria, Madrid, Díaz de Santos.

QUIROGA, V.; y ALONSO, A. (2011): Sueños de bolsillo. Menores migrantes no acompañados en España, Barcelona, Fundación Pere Tarrés.

RODRÍGUEZ CABRERO G. et al. (2011): Servicios sociales y cohesión social, Madrid, Consejo Económico y Social.

SEVILLANO, M. L.; GONZÁLEZ, M. P.; y REY, L. (2009): "Televisión, actitudes y drogas en adolescentes: investigación sobre sus efectos", Comunicar: Revista Científica Iberoamericana de Comunicación y Educación, nํㅜ 33, págs. 185-192.

TEDESCO. J. C. (2011): "Los desafíos de la educación básica en el siglo XXI”, Revista Iberoamericana de Educación, nํㅜ 55, págs. 31-47.

TOBOSO, M. et al. (2012): "Sobre la educación inclusiva en España: políticas y prácticas”, Intersticios: Revista Sociológica de Pensamiento Crítico, vol. 6, no 1, págs. 294-299.

TORRES, J. (2010): La justicia curricular, Madrid, Morata.

UCEDA, F. X.; y PÉREZ, J. V. (2010): “¿Es operativa la intervención pública con los adolescentes en conflicto con la ley?: un análisis desde las trayectorias y los escenarios", Anduli. Revista Andaluza de Ciencias Sociales, no9, págs. 53-69.

UNESCO (2012): Informe de Seguimiento de la EPT en el Mundo. Los jóvenes y las competencias: trabajar con la educación, París, Unesco.

- (2008): La educación inclusiva: el camino hacia el futuro. Documento de referencia, París, Unesco.

- (1994): Conferencia Mundial sobre Necesidades Educativas Especiales: Acceso y Calidad. Declaración de Salamanca y Marco de Acción sobre Necesidades Educativas Especiales, Salamanca, Ministerio de Educación y Ciencia.

UNICEF (2012): La infancia en España, 2012-2013: el impacto de la crisis en las niñas y niños, Madrid, Unicef.

VEGA, A. (2011): La infancia robada. A propósito de los menores vulnerables, Barcelona, Davinci.

VEGA, A.; y ARAMENDI, P. (2012): Drogas en los PCPI: un reto educativo, Vitoria, Gobierno Vasco.

- (2011): “La mediación educativa de los programas de cualificación profesional inicial: a propósito de las drogas", Educación XXI, vol. 14, nํㅡ 2, págs. 213-236.

VEGA, A. et al. (2012): "A la salud de los adolescentes y los jóvenes: notas para el debate sobre las drogas", A tu Salud, no -77, págs. 11-15.

YAGÜE, V. (2012): Alex. Los niños salvajes, Barcelona, Plataforma. 